\title{
Prognostic considerations of the new World Health Organization classification of lung adenocarcinoma
}

\author{
Alain C. Borczuk \\ Affiliation: Weill Cornell Medicine, New York, NY, USA. \\ Correspondence: Alain C. Borczuk, Weill Cornell Medicine, 1300 York Avenue ST10-1000A, New York, NY 10065, \\ USA. E-mail: Alb9003amed.cornell.edu \\ ABSTRACT The 2015 World Health Organization (WHO) lung adenocarcinoma classification divides \\ tumours into categories of indolent pre-invasive, minimally invasive and predominantly lepidic and, by \\ examining predominant patterns of invasion, allows for further stratification into intermediate and high- \\ grade tumours. The impact of the 2015 classification on prognosis was reviewed by a PubMed search for \\ search terms "adenocarcinoma", "lung pathology" and "prognosis" and relevant publications reviewed. \\ These were sorted for data on stage and survival as impacted by histological classification, and survival \\ studies were separated into all stage versus stage 1 studies. Predictive aspects of histological classification \\ were also examined, but molecular correlates were not. The separation of adenocarcinoma in situ and \\ minimally invasive adenocarcinoma from invasive subtypes as distinct prognostic entities and the \\ prognostic significance, for disease specific and overall survival for low- and high-grade categories, are \\ discussed. The impact on stage at presentation including risk of node metastasis by histology is examined, \\ as well as histology in relation to recurrence after surgery. Early data with regard to the value of \\ predominant histology in the prediction of chemotherapy response will also be explored.
}

0 @ERSpublications

Lepidic lung ADC often early stage and indolent; solid and micropapillary tumours often metastatic and recurrent http://ow.ly/DCiT305xExK

\section{Introduction}

The classification of lung adenocarcinoma has undergone significant change in the last 17 years, with evolution in the World Health Organization (WHO) 1999 [1], 2004 [2] and 2015 [3] classification. In 2011, the International Association for the Study of Lung Cancer (IASLC) revised the 2004 classification to incorporate important new elements [4], which were largely adopted in the 2015 classification.

In pathologic classification, stratification, leading to a complex classification, and merging, for simplification, are opposing viewpoints. The 1999 and 2004 classification clarified bronchioloalveolar carcinoma (BAC) by defining it more stringently to include only $100 \%$ noninvasive tumours. The term $\mathrm{BAC}$, from the original Armed Forces Institute of Pathology fascicle as a peripheral adenocarcinoma, was already entrenched and encompassed mucinous and non-mucinous tumours, some invasive and some with lobar consolidation. The merging of these entities under one category of BAC was so well-established that to stratify using a more stringent criterion in 1999 and 2004 was not successful. As a result, the term BAC needed to be eliminated and replaced by terminology that better reflected the modern noninvasive definition. In doing so, the 2011 IASLC recommendation established a progression from pre-invasive adenocarcinoma in situ to overtly invasive adenocarcinoma through two additional categories: a minimally invasive adenocarcinoma with $\leqslant 5.0 \mathrm{~mm}$ invasion and a lepidic predominant adenocarcinoma, in which the

Received: Aug 192016 | Accepted after revision: Oct 232016

Conflict of interest: None declared.

Provenance: Submitted article, peer reviewed.

Copyright OERS 2016. ERR articles are open access and distributed under the terms of the Creative Commons Attribution Non-Commercial Licence 4.0. 
alveolar growth without architectural effacement represented a majority of a tumour but with invasion $>5.0 \mathrm{~mm}$. The expectation was that tumours in these new strata might have better prognosis than their frankly invasive counterparts.

The other side of the 1999/2004 classification, which defined BAC as a noninvasive tumour, was a very broad category of mixed subtype adenocarcinoma ranging from tumours with small amounts of invasion to those with heterogeneous invasive patterns. Only relatively pure single pattern adenocarcinomas were named separately, such as acinar or papillary. A formal cut-off for extent of a single pattern was not delineated, although $>75 \%$ was used by some authors [5]. Without a clear cut-off and with the known heterogeneity of patterns, mixed subtype adenocarcinoma was a merged category dominating adenocarcinoma reports. In the 2011 classification, the finding of a predominant pattern, that is the most common pattern within a tumour, was used to name it as predominantly lepidic, acinar, papillary, micropapillary or solid. This resulted in stratification of the mixed subtype and, even in the most heterogeneous of tumours, forced designation of a predominant pattern (figures 1 and 2).

This tiered classification would be an improvement over the 2004 classification if its granularity had greater biological significance, justifying its use. Studies have led to the conclusion that this approach of assigning predominant pattern leads to reproducible categories [6], so that it is not unreasonably complex, and that these categories subdivide lung adenocarcinoma into prognostically relevant groups. Specifically, this inter-observer ring study revealed that classic patterns of adenocarcinoma were reproducible, and that even difficult examples had a moderate degree of concordance for predominant pattern.

The classification also introduced diagnostic categories into small samples such as biopsy and cytology. While this focused on major categories of carcinoma such as squamous cell versus adenocarcinoma [7], there has been interest in using small samples to predict predominant pattern in the effort to grade adenocarcinoma on such samples. Efforts to do that have been met with only modest agreement with resection specimens among acinar cases, but poor agreement for solid and micropapillary specimens. The most specific criteria, such as papillary structures, may not be present in a large number of samples. Much of this disagreement may be due to sampling, as secondary and tertiary patterns on resection may be
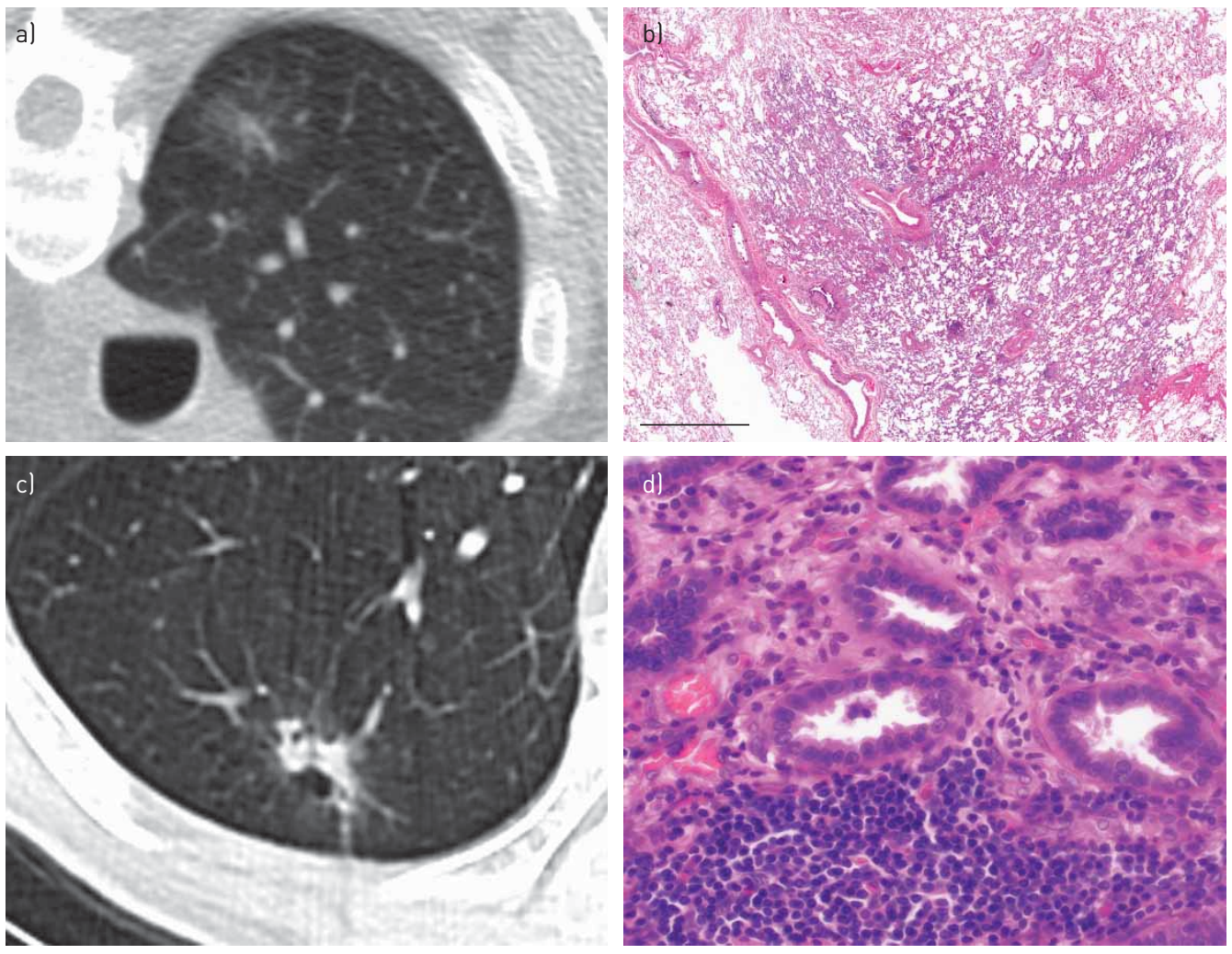

FIGURE 1 Imaging and histological correlation in lung adenocarcinoma classification. a) Computed tomography (CT) scan demonstrating a ground-glass nodule that on resection represents adenocarcinoma in situ. b) The preserved alveolar architecture is seen at low magnification in this adenocarcinoma in situ. Scale bar $=3 \mathrm{~mm}$. c) CT scan demonstrating a solid area surrounded by a rim of ground-glass attenutation. d) On resection, this was an acinar predominant adenocarcinoma with gland formation seen on the histology image. 

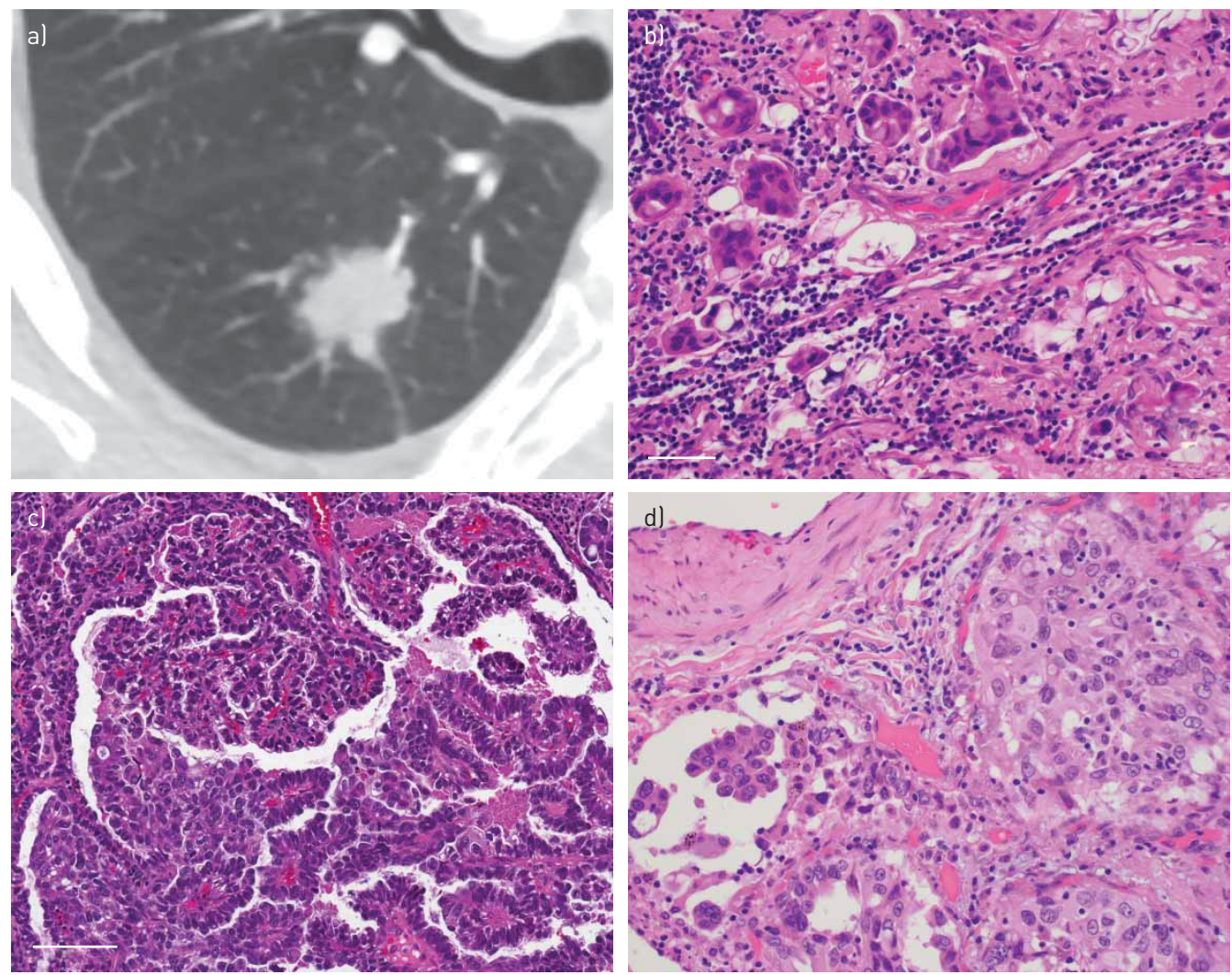

FIGURE 2 Imaging and histological correlation in lung adenocarcinoma classification. al Computed tomography (CT) scan demonstrating a solid attenuation nodule. b) A micropapillary pattern with small nests of tumours cells surrounded by retraction artefact was seen on the resection specimen. Scale bar $=50 \mu \mathrm{m}$. c) Papillary pattern is shown with fibrovascular cores and an arborising architecture lined by neoplastic cells. Scale bar $=100 \mu \mathrm{m}$. d) On the right side a solid tumour nest demonstrating solid pattern adenocarcinoma is seen. A typical feature of lung adenocarcinoma heterogeneity is shown by an adjacent focus on the left showing micropapillary pattern.

overrepresented in a given small sample $[8,9]$. Some promising efforts to grade cytology samples suggest that prognostication may be part of future classifications [10].

The current classification includes adenocarcinoma in situ and minimally invasive adenocarcinoma (both of which can be non-mucinous or mucinous, with mucinous adenocarcinoma in situ and minimally invasive adenocarcinoma being relatively rare tumours), lepidic, acinar, papillary, solid, micropapillary predominant adenocarcinoma and invasive mucinous adenocarcinoma. Studies have addressed the validity of the pre-invasive and minimally invasive categories, examined the impact of predominant pattern on stage, investigated the prognostic role of predominant pattern in node negative adenocarcinoma, and have begun to predict the impact of these prognostic categories on therapeutic approaches.

\section{Prognostic significance of the 1999/2004 classification}

SAKURAI et al. [11] published a large surgical series of 7921 predominantly stage $1 \mathrm{~A}$ and 1B adenocarcinoma, stratified by histological pattern including BAC, acinar, papillary, solid and mixed subtype. This was based upon the WHO 1999/2004 classification, although BAC may not have been purely noninvasive. The BAC group had significantly better 5 -year survival than the acinar, papillary or solid groups; for stage $1 \mathrm{~A}$ this was $95 \%$ for $\mathrm{BAC}$ and $86 \%, 86 \%$ and $74 \%$ for acinar, papillary and solid, respectively. For stage 1B, BAC showed the highest 5 -year survival (87\%) versus acinar, papillary and solid which were $70 \%, 81 \%$ and $62 \%$, respectively. A large proportion were classified as mixed subtype. These mixed subtype tumours had a 5 -year survival of $89 \%$ and $77 \%$ for stage $1 \mathrm{~A}$ and stage $1 \mathrm{~B}$, which was in between BAC and other specific categories.

It is important to frame these observations and subsequent ones in the context of aggregate lung cancer survival data [12]. In a study examining survival in node-negative carcinoma, increasing size was an important determinant of 5-year survival. In tumours $<3.0 \mathrm{~cm} 5$-year survival was reported at $71 \%$ and for tumours $<2.0 \mathrm{~cm}$ at $77 \%$. As tumour size increases to $5.0 \mathrm{~cm}$ 5-year survival drops to $58 \%$, and for 
tumours $>5 \mathrm{~cm}$ but $<7.0 \mathrm{~cm}$ in size it drops to $49 \%$. Very large tumours $(>7.0 \mathrm{~cm})$ have a low 5 -year survival of $35 \%$, even when node negative. As we compare this to adenocarcinoma-specific data from the study by SAKURAI et al. [11], this aggregate data is comparable to that of stage $1 \mathrm{~A}$ for solid adenocarcinoma; notably other adenocarcinoma categories showed higher 5-year survival when compared to this aggregate data.

\section{New WHO classification}

Rates of nodal metastasis

Rates of nodal metastasis vary by predominant pattern. In one study [13], lepidic adenocarcinoma were node positive $7 \%$ of the time, in contrast to papillary, acinar, solid and micropapillary adenocarcinoma which were node positive $43 \%, 47 \%, 51 \%$ and $76 \%$ of the time, respectively. TsuTA et al. [14] reported lymph node-positive rates of $3 \%, 7 \%, 28 \%, 39 \%, 44 \%$ and $39 \%$ in lepidic, invasive mucinous adenocarcinoma, papillary, acinar, solid and micropapillary adenocarcinoma, respectively. Russell et al. [15] found node-positive rates of 0\%, $27 \%, 23 \%, 44 \%$ and $35 \%$ in lepidic, acinar, papillary, micropapillary and solid adenocarcinoma, respectively. While the lowest node-positive rates in lepidic adenocarcinoma were a consistent finding of their early stage, varied rates were seen for other subtypes. While micropapillary had highest rates of node positivity, even within the micropapillary predominant group different studies show a range from $39 \%$ to $82 \%$ [16].

Prognosis across all stages

In 440 Japanese lung adenocarcinoma patients, YoshizAwa et al. [16] showed stage and high grade were significant prognosticators. One caveat is that the high-grade group was compared to the combined low-grade and intermediate-grade group, which included adenocarcinoma in situ and minimally invasive adenocarcinoma cases. Disease free survival (DFS) at 5 years showed that solid and micropapillary adenocarcinoma frequently recurred (all 19 cases of micropapillary adenocarcinoma recurred) and 5-year survival was poorest in these groups. This study supports solid and micropapillary adenocarcinoma as higher stage tumours with higher rates of recurrence. It is of note that while only 14 patients had partial resections, the 5-year survival of the partial resection group was particularly low at 58\%, so that surgical approach may have impacted on survival in some patients. In a similar series, Gu et al. [17] studied 292 stage I-III Chinese lung adenocarcinoma patients. This series had a relatively large number of predominantly micropapillary cases $(n=30)$, which were also of higher stage at presentation. Stage and high grade were linked with DFS and overall survival. Once again, the high-grade category was significant only when analysed in conjunction with the combined intermediate and favourable prognosis group, and the favourable group included 15 cases of minimally invasive adenocarcinoma and adenocarcinoma in situ, as well as 31 cases of lepidic adenocarcinoma to achieve a p-value of 0.038 . Both these studies show high-grade tumour as an independent variable, but are limited by inclusion of adenocarcinoma in situ and minimally invasive adenocarcinoma in their analysis. However, despite this criticism, the survival groups indicate lepidic, acinar/papillary and micropapillary/solid adenocarcinoma as three distinct tiers, a finding also reported by Russell et al. [15].

In a series of 487 stage I-IV adenocarcinoma from Germany, WARTH et al. [13] found that survival of predominant lepidic adenocarcinoma differed from invasive predominant patterns, and micropapillary tumours were of higher stage at presentation. By multivariate analysis, stage and predominant pattern remained significant; this required comparison of a combination of papillary/solid/micropapillary adenocarcinoma compared to lepidic (not including adenocarcinoma in situ or minimally invasive adenocarcinoma tumours). In this study, papillary adenocarcinoma was in the high-grade group. Only predominant pattern contributed to the overall prognosis assessment. In a similar fashion, a series of 486 patients showed prognosis was impacted by tumour size $>3.0 \mathrm{~cm}$, age, lymphovascular invasion and stage $>1$. High-grade mostly solid pattern tumours were a distinct prognosis group from the lower grade group, with lepidic adenocarcinoma combined with acinar and papillary [18]. In a study of 573 patients, solid or micropapillary adenocarcinoma were of higher stage [19]. Stage was the most important survival predictor, but micropapillary and solid pattern had lower disease-specific survival (DSS). In their analysis, lepidic adenocarcinoma was grouped with acinar/papillary. These studies did not include adenocarcinoma in situ and minimally invasive adenocarcinoma in their low-grade group.

The grouping of lepidic adenocarcinoma with the intermediate group to contrast the high-grade group for statistical significance underscores the prognostic importance of the lepidic predominant adenocarcinoma as an early stage indolent tumour in the 2011 IASLC classification [20]. The favourable prognosis of the predominant lepidic pattern tumour is a major component of the prognostic classification.

In a series of 407 adenocarcinomas from France [21], high stage had adverse survival impact, as did high grade (mostly solid adenocarcinoma). In a study of 904 patients published by TsuTA et al. [14], stage was of critical importance and 5- and 10-year overall survival followed the predominant histology; this group also shows a contribution of lymphovascular invasion to adverse prognosis of T1 patients. 
Not every series has confirmed the prognostic significance of the classification. In a series of 294 cases from Iceland [22], overall survival was not impacted by predominant histology, except potentially in the subset of lepidic adenocarcinoma. There were few adenocarcinoma in situ/minimally invasive adenocarcinoma tumours and very few $(n=3)$ micropapillary tumours in this study.

In summary, lepidic adenocarcinomas were of a lower stage and showed better prognosis while micropapillary/ solid tumours were of a higher stage with poorer prognosis when compared to categories including lepidic adenocarcinoma. While acinar and papillary adenocarcinoma were generally in an intermediate group, the effect of these patterns on survival was less predictable. These findings are summarised in table 1.

\section{The significance of adenocarcinoma in situ and minimally invasive adenocarcinoma}

YoshizAwa et al. [16] showed 100\% DFS for adenocarcinoma in situ and minimally invasive adenocarcinoma with $100 \%$ overall survival confirmed by TsuTA et al. [14], with very high overall survival of $>95 \%$ being reported at 12 years. T1 prognosis was impacted by removal of adenocarcinoma in situ/minimally invasive adenocarcinoma cases, supporting the separation of cases as $\mathrm{T}_{\mathrm{AIS}}$ or $\mathrm{T}_{\mathrm{MIA}}$. Node metastasis was not seen in adenocarcinoma in situ/minimally invasive adenocarcinoma [15], adenocarcinoma in situ and minimally invasive adenocarcinoma cases showed no disease-related deaths [20] and adenocarcinoma in situ/minimally invasive adenocarcinoma patients had 100\% DFS at 5 years [25].

\section{Recurrence versus survival}

In a series of 354 patients lepidic versus non-lepidic tumours showed a significant difference for DFS but not for DSS [20]. The number of micropapillary predominant tumours was low. Since differences in DFS were significant, it is possible that the high number of sublobar resections in this study (45\%) resulted in a higher than expected proportion of local recurrence. Since DSS was not impacted, recurrences may have been successfully treated. The study by Hung et al. [19] also indicates a higher rate of recurrence with micropapillary or solid adenocarcinoma.

Among 177 stage 1 patients (all T1a N0) the 5-year DFS was 95\%, 92\%, 83\%, 67\% and 58\% for lepidic, papillary, acinar, solid and micropapillary adenocarcinoma, respectively. Increased post-operative recurrence for the high-risk histology group was observed despite all patients undergoing lobectomy and node dissection [26].

\section{Stage 1 adenocarcinoma}

In T1a N0 $(<2.0 \mathrm{~cm})$ adenocarcinomas [27], three groups emerged: lepidic, acinar/papillary and solid/ micropapillary. High 3-year overall survival in the first two groups (98\% and 97\%, respectively) decreased to $85 \%$ in the high-risk group. Recurrence-free survival was high in group 1, 88\% in group 2 and $64 \%$ in group 3. Failure rate in group 3 was particularly high when compared to other studies. One potential explanation is that wedge and sublobar resections were performed in 92 patients, including $45 \%$ of the high-risk group and $38 \%$ of the intermediate group.

In a series of 283 stage 1 adenocarcinomas [23], Overall survival for lepidic adenocarcinoma was better than for the other patterns. Recurrence was highest for micropapillary or solid types and lowest for lepidic adenocarcinoma. Pleural invasion, age and high grade were all risks for recurrence, but overall survival difference was only seen for lepidic adenocarcinomas.

TABLE 1 Studies of predominant histology and outcome: mixed stages

\begin{tabular}{|c|c|c|c|c|c|c|c|c|c|c|c|c|c|c|c|c|c|c|}
\hline \multirow[t]{3}{*}{ First author [ref.] } & \multicolumn{18}{|c|}{ 5-year survival } \\
\hline & \multicolumn{3}{|c|}{ AIS/MIA } & \multicolumn{3}{|c|}{ Lepidic } & \multicolumn{3}{|c|}{ Acinar } & \multicolumn{3}{|c|}{ Papillary } & \multicolumn{3}{|c|}{ Solid } & \multicolumn{3}{|c|}{ Micropapillary } \\
\hline & DFS & DSS & OS & DFS & DSS & OS & DFS & DSS & OS & DFS & DSS & OS & DFS & DSS & OS & DFS & DSS & OS \\
\hline Murakami [20] & 100 & & & 99 & & & 81 & & & 82 & & & 74 & & & 33 & & \\
\hline Hung [23] ${ }^{\#}$ & & & & 80 & 90 & 90 & 70 & 90 & 70 & 70 & 90 & 70 & 40 & 70 & 60 & 50 & 70 & 50 \\
\hline TSAO $[24]^{\#, \pi}$ & & & & 60 & & 60 & 60 & & 55 & 60 & & 55 & 35 & & 45 & 35 & & 45 \\
\hline RusseLL [15] & & & & & & 86 & & & 68 & & & 71 & & & 39 & & & 38 \\
\hline WARTH $_{\text {A13] }}$ & & & & 73 & 80 & 79 & 62 & 79 & 67 & 35 & 56 & 49 & 51 & 66 & 58 & 34 & 60 & 45 \\
\hline Gu [17] & 100 & & 100 & 72 & & 91 & 54 & & 72 & 56 & & 72 & 46 & & 58 & 25 & & 46 \\
\hline
\end{tabular}

AIS: adenocarcinoma in situ; MIA: minimally invasive adenocarcinoma; DFS: disease-free survival; DSS: disease-specific survival; OS: overall survival. \# : data estimated from Kaplan-Meier curves; ${ }^{\text {ๆ: }}$ papillary/acinar combined, solid/micropapillary combined. 
In 2011, Yoshizawa [25] examined a set of stage 1 adenocarcinomas using the predominant pattern approach. DFS was $100 \%$ for adenocarcinoma in situ/minimally invasive adenocarcinoma, $90 \%$ for lepidic, $84 \%$ for acinar, $83 \%$ for papillary, $67 \%$ for micropapillary and $70 \%$ for solid adenocarcinomas. Micropapillary case numbers were low. Necrosis, size and sex were associated with survival and high grade was poorer than the combined intermediate and low grade. Differences in overall survival were not seen.

DuHig et al. [28] examined 145 stage I adenocarcinomas and found that lepidic adenocarcinoma had 100\% survival, with survival for acinar/papillary and solid adenocarcinomas of $70 \%$ and $55 \%$, respectively. These authors concluded that mitotic count was the best stratifier, even within solid, acinar and papillary subtypes.

The stage 1 studies support three tiers as did the mixed stage studies. These findings are summarised in table 2. However, since micropapillary tumours are usually high stage, stage 1 studies have a relative paucity of micropapillary tumours. Recurrence risk was greater for high grade groups, but difference in overall survival was either not observed or reserved for the lepidic adenocarcinoma group.

\section{Margins in limited resections}

NITADORI et al. [29] found that, in addition to the assessment of predominant pattern, the presence of $\geqslant 5 \%$ micropapillary pattern was associated with recurrence in limited resections with margins of $\leqslant 1.0 \mathrm{~cm}$. With margins $>1.0 \mathrm{~cm}$, recurrence rate was comparable to lobectomy. Micropapillary tumours treated by lobectomy showed no difference in recurrence based on micropapillary pattern percentage. In a separate study of sublobar resections compared to lobectomy, predominant histological pattern did not predict recurrence. Survival was best for adenocarcinoma in situ/minimally invasive adenocarcinoma/lepidic and worst for solid type [30]. Optimal margin distance was not assessed.

\section{Predominant pattern and treatment decisions}

An interesting observation by Hung et al. [19] was the association of solid adenocarcinoma with adverse outcome despite adjuvant therapy, raising the possibility that solid adenocarcinoma are relatively resistant to chemotherapy. Given prior publication of KRAS mutation frequency in this subgroup [31], it is possible that a combination of solid pattern and KRAS mutation are linked to diminished response; however, KRAS mutation impact on chemotherapy response and survival remain controversial [32].

TSAO et al. [24] examined a single slide from 582 patients with stage I-III adenocarcinoma, with 44\% of their cohort in stage II or greater. The lepidic adenocarcinomas were excluded from treatment-related analyses due to paucity of cases, but the few micropapillary adenocarcinomas were retained. Consistent with other studies, increased rate of recurrence in micropapillary and solid adenocarcinoma was seen, but no difference was seen in overall survival. Importantly, potential differences in treatment response based on predominant histology were seen, suggesting that adjuvant therapy may be less effective or require different selection algorithms with some histological patterns. This is a confirmation of the observation of Hung et al. [19].

A recent study [33] examined 928 patients with stage IB adenocarcinoma. The overall survival in this cohort was $87 \%$ and DFS was $73 \%$. However, only six patients had lepidic adenocarcinoma and only 12 had micropapillary adenocarcinoma. No deaths were reported in the lepidic group. Solid versus acinar/papillary adenocarcinoma was the most significant comparison for DFS and overall survival. Adjuvant chemotherapy impacted recurrence-free survival but not overall survival. Interestingly, solid adenocarcinoma has the greatest chemotherapy benefit and acinar/papillary adenocarcinoma showed no benefit. This finding was not consistent with that of TsaO et al. [24]. Treatment type, handling of recurrences and rates of epidermal growth factor receptor targeting therapy after recurrence were not

TABLE 2 Studies of predominant histology and outcome: stage I only

First author [ref.]

5-year survival

\begin{tabular}{|c|c|c|c|c|c|c|c|c|c|c|c|c|}
\hline & \multicolumn{2}{|c|}{ AIS/MIA } & \multicolumn{2}{|c|}{ Lepidic } & \multicolumn{2}{|c|}{ Acinar } & \multicolumn{2}{|c|}{ Papillary } & \multicolumn{2}{|c|}{ Solid } & \multicolumn{2}{|c|}{ Micropapillary } \\
\hline & DFS & OS & DFS & OS & DFS & OS & DFS & OS & DFS & OS & DFS & OS \\
\hline$Y_{\text {ANG }}[26]$ & & & 95 & & & 83 & & 92 & & 67 & & 58 \\
\hline YoshizaWA [25] & 100 & 74 & 90 & 75 & 84 & 75 & 83 & 75 & 70 & 61 & 67 & 61 \\
\hline Hung $[23]^{+}$ & & & $>90$ & 100 & 80 & 80 & 80 & 80 & 60 & 75 & 60 & NA \\
\hline
\end{tabular}

AIS: adenocarcinoma in situ; MIA: minimally invasive adenocarcinoma; DFS: disease-free survival; OS: overall survival; NA: not available. ${ }^{\text {: }}$ papillary/acinar combined, solid/micropapillary combined; ๆ : 3-year survival; ${ }^{+}$: data estimated from Kaplan-Meier curves. 
reported and could account for inter-study inconsistency. In addition, this group of stage IB patients contrasted those of TSAO et al. [24], who reported a high proportion of stage 2 and 3 patients. Since adjuvant therapy is not currently offered to all stage IB patients, these differences, based on histology alone, need resolution with further study, including what may represent differences in driver mutations among Western and Eastern patient cohorts. It also suggests that treatment effect may need study within, not across, stage categories.

\section{Conclusion}

The 2015 WHO classification of adenocarcinoma has prognostic significance. Adenocarcinoma in situl minimally invasive adenocarcinoma have high disease-specific survival and are not associated with metastasis. Lepidic adenocarcinoma have low node metastatic rate and thus present at early stage; they are generally associated with favourable recurrence-free survival and DSS. The other invasive patterns seem to stratify with acinar/papillary adenocarcinoma as an intermediate group with a higher rate of node metastasis at presentation than lepidic adenocarcinoma, and solid/micropapillary with highest recurrence rate among adenocarcinomas, showing lowest DSS and lowest overall survival.

\section{References}

Travis WD, Colby TV, Corrin B, et al. Histological Typing of Lung and Pleural Tumours. Germany, Springer, 1999. Travis WD, Brambilla E, Muller-Hermelink HK, et al. Pathology and Genetics Tumours of the Lung, Pleura, Thymus and Heart. Lyon, IARC Press, 2004.

3 International Agency for Research on Cancer. WHO Classification of Tumours of the Lung, Pleura, Thymus and Heart. Geneva, World Health Organization, 2015.

4 Travis WD, Brambilla E, Noguchi M, et al. International Association for the Study of Lung Cancer/American Thoracic Society/European Respiratory Society international multidisciplinary classification of lung adenocarcinoma. J Thorac Oncol 2011; 6: 244-285.

5 Silver SA, Askin FB. True papillary carcinoma of the lung: a distinct clinicopathologic entity. Am J Surg Pathol 1997; 21: 43-51.

6 Thunnissen E, Beasley MB, Borczuk AC, et al. Reproducibility of histopathological subtypes and invasion in pulmonary adenocarcinoma. An international interobserver study. Mod Pathol 2012; 25: 1574-1583.

7 Rekhtman N, Ang DC, Sima CS, et al. Immunohistochemical algorithm for differentiation of lung adenocarcinoma and squamous cell carcinoma based on large series of whole-tissue sections with validation in small specimens. Mod Pathol 2011; 24: 1348-1359.

8 Rodriguez EF, Dacic S, Pantanowitz L, et al. Cytopathology of pulmonary adenocarcinoma with a single histological pattern using the proposed International Association for the Study of Lung Cancer/American Thoracic Society/European Respiratory Society (IASLC/ATS/ERS) classification. Cancer Cytopathol 2015; 123: 306-317.

9 Rudomina DE, Lin O, Moreira AL. Cytologic diagnosis of pulmonary adenocarcinoma with micropapillary pattern: does it correlate with the histologic findings? Diagn Cytopathol 2009; 37: 333-339.

10 Sigel CS, Rudomina DE, Sima CS, et al. Predicting pulmonary adenocarcinoma outcome based on a cytology grading system. Cancer Cytopathol 2012; 120: 35-43.

11 Sakurai H, Asamura H, Miyaoka E, et al. Differences in the prognosis of resected lung adenocarcinoma according to the histological subtype: a retrospective analysis of Japanese lung cancer registry data. Eur J Cardiothorac Surg 2014; 45: 100-107.

12 Rami-Porta R, Ball D, Crowley J, et al. The IASLC lung cancer staging project: proposals for the revision of the T descriptors in the forthcoming (seventh) edition of the TNM classification for lung cancer. J Thorac Oncol 2007; 2: 593-602.

13 Warth A, Muley T, Meister M, et al. The novel histologic International Association for the Study of Lung Cancer/ American Thoracic Society/European Respiratory Society classification system of lung adenocarcinoma is a stage-independent predictor of survival. J Clin Oncol 2012; 30: 1438-1446.

14 Tsuta K, Kawago M, Inoue E, et al. The utility of the proposed IASLC/ATS/ERS lung adenocarcinoma subtypes for disease prognosis and correlation of driver gene alterations. Lung Cancer 2013; 81: 371-376.

15 Russell PA, Wainer Z, Wright GM, et al. Does lung adenocarcinoma subtype predict patient survival? A clinicopathologic study based on the new International Association for the Study of Lung Cancer/American Thoracic Society/European Respiratory Society international multidisciplinary lung adenocarcinoma classification. J Thorac Oncol 2011; 6: 1496-1504.

16 Yoshizawa A, Sumiyoshi S, Sonobe M, et al. Validation of the IASLC/ATS/ERS lung adenocarcinoma classification for prognosis and association with EGFR and KRAS gene mutations: analysis of 440 Japanese patients. $J$ Thorac Oncol 2013; 8: 52-61.

$17 \mathrm{Gu}$ J, Lu C, Guo J, et al. Prognostic significance of the IASLC/ATS/ERS classification in Chinese patients: a single institution retrospective study of 292 lung adenocarcinoma. J Surg Oncol 2013; 107: 474-480.

18 Yanagawa N, Shiono S, Abiko M, et al. The correlation of the International Association for the Study of Lung Cancer (IASLC)/American Thoracic Society (ATS)/European Respiratory Society (ERS) classification with prognosis and EGFR mutation in lung adenocarcinoma. Ann Thorac Surg 2014; 98: 453-458.

19 Hung JJ, Yeh YC, Jeng WJ, et al. Predictive value of the International Association for the Study of Lung Cancer/ American Thoracic Society/European Respiratory Society classification of lung adenocarcinoma in tumor recurrence and patient survival. J Clin Oncol 2014; 32: 2357-2364.

20 Murakami S, Ito $\mathrm{H}$, Tsubokawa N, et al. Prognostic value of the new IASLC/ATS/ERS classification of clinical stage IA lung adenocarcinoma. Lung Cancer 2015; 90: 199-204.

21 Mansuet-Lupo A, Bobbio A, Blons $\mathrm{H}$, et al. The new histologic classification of lung primary adenocarcinoma subtypes is a reliable prognostic marker and identifies tumors with different mutation status: the experience of a French cohort. Chest 2014; 146: 633-643. 
22 Oskarsdottir GN, Bjornsson J, Jonsson S, et al. Primary adenocarcinoma of the lung - histological subtypes and outcome after surgery: using the IASLC/ATS/ERS classification of lung adenocarcinoma. APMIS 2016; 124: 384-392.

23 Hung JJ, Jeng WJ, Chou TY, et al. Prognostic value of the new International Association for the Study of Lung Cancer/American Thoracic Society/European Respiratory Society lung adenocarcinoma classification on death and recurrence in completely resected stage I lung adenocarcinoma. Ann Surg 2013; 258: 1079-1086.

24 Tsao MS, Marguet S, Le Teuff G, et al. Subtype classification of lung adenocarcinoma predicts benefit from adjuvant chemotherapy in patients undergoing complete resection. J Clin Oncol 2015; 33: 3439-3446.

25 Yoshizawa A, Motoi N, Riely GJ, et al. Impact of proposed IASLC/ATS/ERS classification of lung adenocarcinoma: prognostic subgroups and implications for further revision of staging based on analysis of 514 stage I cases. Mod Pathol 2011; 24: 653-664.

26 Yang F, Chen K, Liao Y, et al. Risk factors of recurrence for resected TlaN0M0 invasive lung adenocarcinoma: a clinicopathologic study of 177 patients. World J Surg Oncol 2014; 12: 285.

27 Yoshiya T, Mimae T, Tsutani Y, et al. Prognostic role of subtype classification in small-sized pathologic N0 invasive lung adenocarcinoma. Ann Thorac Surg 2016; 102: 1668-1673.

28 Duhig EE, Dettrick A, Godbolt DB, et al. Mitosis trumps T stage and proposed International Association for the Study of Lung Cancer/American Thoracic Society/European Respiratory Society classification for prognostic value in resected stage 1 lung adenocarcinoma. J Thorac Oncol 2015; 10: 673-681.

29 Nitadori J, Bograd AJ, Kadota K, et al. Impact of micropapillary histologic subtype in selecting limited resection vs lobectomy for lung adenocarcinoma of $2 \mathrm{~cm}$ or smaller. J Natl Cancer Inst 2013; 105: 1212-1220.

30 Dembitzer FR, Flores RM, Parides MK, et al. Impact of histologic subtyping on outcome in lobar vs sublobar resections for lung cancer: a pilot study. Chest 2014; 146: 175-181.

31 Rekhtman N, Ang DC, Riely GJ, et al. KRAS mutations are associated with solid growth pattern and tumor-infiltrating leukocytes in lung adenocarcinoma. Mod Pathol 2013; 26: 1307-1319.

32 Yu HA, Sima CS, Shen R, et al. Prognostic impact of KRAS mutation subtypes in 677 patients with metastatic lung adenocarcinomas. J Thorac Oncol 2015; 10: 431-437.

33 Luo J, Huang Q, Wang R, et al. Prognostic and predictive value of the novel classification of lung adenocarcinoma in patients with stage IB. J Cancer Res Clin Oncol 2016; 142: 2031-2040. 